\title{
Prevalencia de síndrome metabólico y factores de riesgo cardiovascular en el personal masculino de la empresa eléctrica de Azogues C.A .
}

\section{Prevalence of metabolic syndrome and cardiovascular risk factors in the male staff of the electricity company of Azogues C.A.}

Karla Priscilla Suárez Morquecho. ${ }^{1}$ \& Mónica Patricia Vinueza Alvear. ${ }^{2}$

Recibido: 11-03-2017 / Revisado: 12-05-2017 Aceptado: 18-06-2018/ Publicado: 01-07-2018

\section{Abstract.}

DOI: https://doi.org/10.33262/cienciadigital.v2i3.149

This article focused on Metabolic Syndrome (MS) which is a cluster of conditions that includes excess central obesity, high blood pressure and fasting glucose levels, hypertriglyceridemia and decreased HDL cholesterol levels. It is a growing public health problem in Ecuador and it is associated with the development of cardiovascular diseases, hypertension and diabetes mellitus, which are considered to be the main causes of death in this country. Its etiology is a set of risk factors that commonly appear together being the work environment and lifestyle the preeminent factors in its appearance. In order to estimate its prevalence and identify the associated factors a study was applied to employees of Empresa Eléctrica de Azogues.

Methods: a cross-sectional descriptive study in which 114 male workers were used as sample from whom laboratory-tests (basal glucose, HDL and triglycerides) were supplied by the company's medical department. These were analyzed, as well as blood pressure and anthropometric data were evaluated: weight, height, abdominal perimeter. In order to assess eating-habits, physical activity, alcohol and tobacco consumption, as well as pathological background a survey was applied. The data gathered throughout this survey was done by means of SPSS V20- Statistical Package for the Social Sciences.

\footnotetext{
${ }_{1}^{1}$ Médico General, Cañar, Ecuador, karlita-suarezmm@outlook.ec

2 Unidad Técnica de Patología Clínica, Cuenca, Ecuador, karlita-suarezmm@outlook.ec
} 
Results: these tests revealed that the prevalence of MS was $32.5 \%$, being more frequent in adults aged between 40 and 49 years old. 54.05\% of people with MS were overweight and $40.54 \%$ were obese. The presence of abdominal obesity was identified in $100 \%$ of individuals with MS, $94.59 \%$ of whom presented hypertriglyceridemia, which together with low HDL-c levels (89.18\%) were the most predominant criteria in patients with this diagnosis. Statistical significance was found between the consumption of alcohol and high serum triglycerides in the population.

Conclusion: The prevalence of MS was alike to other national studies. There was a statistically significant association between increased age and the development of MS, as well as alcohol consumption and hypertriglyceridemia.

Keywords: Metabolic Syndrome, Overweight, Obesity, Alcohol.

\section{Resumen.}

El Síndrome Metabólico (SM) es un conjunto de trastornos que incluye obesidad central, valores elevados de presión arterial y de glucosa en ayunas, hipertrigliceridemia y concentraciones disminuidas de colesterol HDL. Es un creciente problema de salud pública en Ecuador y está asociado con el desarrollo enfermedades cardiovasculares, hipertensión arterial y diabetes mellitus que constituyen las principales causas de muerte en el país. Su etiología es multifactorial siendo el ambiente laboral y el estilo de vida factores preponderantes en su aparición. Se realizó un estudio para determinar su prevalencia e identificar factores asociados en el personal de la Empresa Eléctrica de Azogues.

Métodos: Estudio descriptivo transversal que incluyó 114 trabajadores masculinos de quienes se analizó exámenes de laboratorio (glucosa basal, HDL y triglicéridos) obtenidos de las historias clínicas del departamento médico de la empresa y se realizó valoración de presión arterial y datos antropométricos: peso, talla, perímetro abdominal. Se aplicó una encuesta para evaluar hábitos alimenticios, actividad física, consumo de alcohol y tabaco, así como antecedentes patológicos. Los datos se procesaron en SPSS V20.

Resultados: la prevalencia de SM fue del 32.5\%, siendo más frecuente en la población de 40 y 49 años. El 54,05\% de personas con SM presentaron sobrepeso y el $40,54 \%$ obesidad. Se identificó la presencia de obesidad abdominal en el $100 \%$ de individuos con SM, 94.59\% de los cuales presentó hipertrigliceridemia, que junto con niveles de c-HDL bajo (89.18\%) fueron los criterios más prevalentes en los pacientes con este diagnóstico. Se encontró significancia estadística entre el consumo de alcohol y triglicéridos séricos elevados en la población. 
CONCLUSIÓN: La prevalencia del SM fue similar a otros estudios nacionales. Se evidenció asociación estadísticamente significativa entre incremento de edad y el desarrollo de SM así como consumo de alcohol e hipertrigliceridemia.

Palabras Claves: Síndrome Metabólico, Sobrepeso, Obesidad, Alcohol.

\section{Introducción.}

El Síndrome Metabólico constituye un conjunto de trastornos caracterizados por la presencia de resistencia a la insulina e hiperinsulinismo, asociados con trastornos del metabolismo de los carbohidratos (hiperglucemia) y de los lípidos (hipertrigliceridemia y disminución del colesterol de alta densidad: HDL), así como presión arterial elevada y obesidad abdominal. El debut de estos trastornos puede ser simultáneo o secuencial en un mismo individuo, y pueden ser causados por factores genéticos y ambientales asociados al estilo de vida, incrementándose de forma significativa el riesgo de padecer diabetes, enfermedad coronaria y enfermedad cerebrovascular (1).

Todos estos factores de riesgo cardiovascular corresponden a características biológicas o del comportamiento presentes en una persona aparentemente sana que están relacionadas en forma independiente con el desarrollo posterior de una enfermedad cardiovascular, la cual puede manifestarse en un período de tiempo determinado, generalmente de 5 a 10 años, pero en múltiples ocasiones el SM no tiene un diagnóstico oportuno como tal, sino más bien en la clínica el diagnóstico llega de forma tardía, tras manifestarse patologías cerebrovasculares, que representan únicamente la punta del iceberg que oculta un sinnúmero de casos de personas que presentan SM (1).

Dentro de los factores modificables que intervienen en la génesis del SM se encuentran la hipertensión arterial, tabaquismo, diabetes, dislipidemias y sedentarismo, los cuales tienen relación con la actividad laboral y rutina sedentaria del individuo así como alimentación inadecuada, malos hábitos del estilo de vida moderno que influyen en el desarrollo de este síndrome.

Se debe considerar que la mayoría de las personas laboralmente activas emplean gran parte de su día en el lugar de trabajo, que se convierte en un ambiente importante para el desarrollo de un estilo de vida muy poco saludable que engloba actividades sedentarias con reducción del esfuerzo físico para la realización de las tareas como permanecer sentados tras un escritorio largas jornadas del día en actividades rutinarias o para el empleo de tecnologías de la información, así también conductas tóxicas como el consumo de alcohol y tabaco (hábitos que en nuestro medio suelen ser incitados por la presión social), siendo todos estos importantes factores de riesgo cardiovascular, por lo que es muy importante 
generar ambientes de trabajo saludables para tratar de minimizarlos, los cuales, si bien están contemplados en la legislación ecuatoriana, su cumplimiento no es considerado por todas las organizaciones laborales en nuestro medio (2).

A nivel laboral, se debe considerar que la vigilancia de la salud de los trabajadores es una obligación de los servicios médicos de empresas, por lo que es muy significativa la labor de identificar la población que presente los distintos factores de riesgo para padecer SM, y proporcionar educación en salud tendiente a lograr estilo de vida saludables en los trabajadores, ya que un diagnóstico temprano de esta afección permitirá aplicar intervenciones precoces para propiciar cambios a estilos de vida saludables, así como tratamientos preventivos que eviten el desarrollo de morbilidades asociadas a este síndrome $(1,3)$.

Si bien existen varios consensos generados para el diagnóstico de SM que incluyen diversas variables y puntos de corte, se ha considerado al de International Diabetes Federation, el mismo que engloba diferencias étnicas de los distintos grupos poblacionales para el punto de corte de la obesidad abdominal siendo considerado criterio preponderante para el diagnóstico de SM considerado referencia para los latinoamericanos $\geq 90 \mathrm{~cm}$ para hombres $\mathrm{y} \geq 80 \mathrm{~cm}$ para mujeres más dos de los siguientes criterios hipertrigliceridemia $\geq$ $150 \mathrm{mg} / \mathrm{dl}$, colesterol de baja densidad HDL < 40mg/dl en el varón y en la mujer <50mg/dl, presión arterial elevada $\geq 130 / 85 \mathrm{mmHg}$ y glicemia $\geq 100 \mathrm{mg} / \mathrm{dl}$ incluyendo diabetes mellitus $(19,20)$.

\section{Materiales y método.}

Se trata de un estudio epidemiológico descriptivo de corte transversal, el universo de estudio será todo el personal masculino que labora en la Empresa Eléctrica de Azogues C.A, que firme el consentimiento informado y la encuesta aceptando así su participación en la presente investigación.

Para la recolección de datos se procedió inicialmente a la aplicación de una encuesta para la evaluación de hábitos alimenticios, actividad física, consumo de tabaco, alcohol así como antecedentes patológicos, además se realizó un examen clínico para la determinación de la presión arterial y medias antropométricas: peso, talla y perímetro abdominal; se analizó exámenes de laboratorio (glucosa basal, colesterol HDL y triglicéridos) obtenidos de las historias clínicas del personal en el departamento médico de la empresa.

Los datos fueron introducidos en una base de datos Excel y posteriormente se procesaron en el programa SPSS 20 en el cual se realizó el análisis estadístico de los mismos mediante la creación de herramientas gráficas (cajas y bigotes, histogramas, etc.) y para determinar la asociación estadística de las variables asociadas a factores de riesgo cardiovascular asociados al estilo de vida (mala alimentación, falta de actividad física, consumo de alcohol y tabaco y actividad laboral sedentaria) y SM se utilizó Chi cuadrado con un IC de 95\%, 
empleando un valor $\mathrm{p}<0,05$ para la significancia de los resultados, por lo cual todas las variables tuvieron que ser trasformadas a cualitativas a través de la dicotomización.

\section{Resultados}

Se analizó una población total de 114 personas que corresponden al personal masculino que labora en la Empresa Eléctrica de Azogues C.A de entre 23 y 64 años (edad promedio 41.58, la mediana 41 y la moda 42 años).

Al aplicar los criterios de la IDF para el diagnóstico de Síndrome Metabólico se encontró una prevalencia en el personal masculino de la Empresa Eléctrica de Azogues C.A, de $32.5 \%$ correspondiente a 37 casos (Tabla Nº 1$)$.

TABLA No 1. Prevalencia de Síndrome Metabólico en el personal masculino de la Empresa Eléctrica de Azogues C.A.

\begin{tabular}{ccc}
\hline \multicolumn{3}{c}{ SÍNDROME METABÓLICO } \\
\hline & Frecuencia & Porcentaje \\
SI & 37 & $32,46 \%$ \\
NO & 77 & $67,54 \%$ \\
TOTAL & 114 & $100 \%$ \\
\hline
\end{tabular}

Fuente: Base de Datos, Personal Masculino, Empresa Eléctrica de Azogues C.A.

En la distribución por grupos de edad se observó que la mayoría de los casos de SM se encontraron entre los 40-49 años (54.05\%), correspondiente a 20 personas, seguido del grupo de 30-39 años (21\%). El grupo con menos casos fue el de 60-69 años que representó $2.6 \%$ con un solo caso (Tabla $\mathrm{N}^{\mathrm{o}} 2$ ).

TABLA N². Distribución del personal con síndrome metabólico por grupo etario.

\begin{tabular}{ccc}
\hline EDAD & \multicolumn{2}{c}{ FRECUENCIA PORCENTAJE } \\
\hline $\mathbf{2 0 - 2 9}$ años & 2 & $5,41 \%$ \\
$\mathbf{3 0 - 3 9}$ años & 8 & $21,62 \%$ \\
$\mathbf{4 0 - 4 9}$ años & 20 & $54,05 \%$ \\
$\mathbf{5 0 - 5 9}$ años & 6 & $16,22 \%$ \\
$\mathbf{6 0 - 6 9}$ años & 1 & $2,70 \%$ \\
Total & 37 & $100 \%$ \\
\hline
\end{tabular}

Fuente: Base de Datos, Personal Masculino, Empresa Eléctrica de Azogues C.A. 
Al aplicar chi cuadrado, se pudo determinar que existía una asociación entre la edad y síndrome metabólico con una significancia estadística del 0.01

Analizando los componentes de SM de forma individual en la población en la que se obtuvo este diagnóstico se estableció que el 5.41\% tenían un IMC normal, 54.05\% sobrepeso, 32.43\% obesidad I, y obesidad II un $8.11 \%$ (Tabla $\mathrm{N}^{\mathrm{o}} 3$ ).

Tabla $\mathbf{N}^{\mathbf{0}}$ 3. Distribución del personal con síndrome metabólico de acuerdo a antropometría: Índice de Masa Corporal.

\begin{tabular}{ccc}
\hline $\begin{array}{c}\text { INDICE DE MASA } \\
\text { CORPORAL }\end{array}$ & FRECUENCIA PORCENTAJE \\
\hline $\begin{array}{c}\text { Normal: 18,50-24.99 } \\
\text { Sobrepeso: } 25,00- \\
\mathbf{2 9 , 9 9}\end{array}$ & 20 & $54,05 \%$ \\
$\begin{array}{c}\text { Obesidad tipo I: } \\
\text { 30,00-34,99 }\end{array}$ & 12 & $32,43 \%$ \\
$\begin{array}{c}\text { Obesidad tipo II: } \\
\mathbf{3 5 , 0 0 - 3 9 , 0 0}\end{array}$ & 3 & $8.11 \%$ \\
\hline Total & 37 & $100 \%$ \\
\hline
\end{tabular}

Fuente: Base de Datos, Personal Masculino, Empresa Eléctrica de Azogues C.A.

En cambio, toda la población diagnosticada con SM tenía criterio positivo para perímetro abdominal (100\%), requisito indispensable para su diagnóstico según los enunciados de IDF, seguido por la hipertrigliceridemia con un $94.59 \%$, valores bajos de c-HDL se registró en el $89.18 \%$. La presión arterial se encontró elevada en 22 personas mientras que el criterio que

se presentó en menor porcentaje en este grupo fue la glicemia mayor o igual a $100 \mathrm{mg} / \mathrm{dl}$ con $18.92 \%$ (Tabla No 4 ).

TABLA No 4. Porcentaje de indicadores de riesgo según criterios IDF en el personal masculino con síndrome metabólico de la Empresa Eléctrica de Azogues C.A.

\begin{tabular}{ccc}
\hline CRITERIOS & $\mathbf{N}^{\circ}$ CASOS PORCENTAJE \\
\hline Perímetro Abdominal & 37 & $100 \%$ \\
Colesterol-HDL & 33 & $89.18 \%$ \\
Triglicéridos & 35 & $94.59 \%$ \\
Presión Arterial & 22 & $59,45 \%$ \\
Glicemia & 7 & $18.92 \%$ \\
\hline
\end{tabular}

Fuente: Base de Datos, Personal Masculino, Empresa Eléctrica de Azogues C.A. 
Al valorar el gasto de energía en relación a la función laboral se estimó que la mayor prevalencia de síndrome metabólico fue para aquellos cargos con escasa actividad laboral, como es el caso de choferes y administrativos con un $56.76 \%$, seguidos de aquellos que cumples funciones que demandan abundante actividad (liniero y lector) considerada como funciones que demandan abundante actividad (27.03\%), el menor porcentaje se registró en técnicos y auxiliares los cuales desempeñaban activad laboral con gasto energético moderado con un porcentaje del 16.22\%, sin embargo, no se encontró asociación estadísticamente significativa entre estas variables (Tabla $\mathrm{N}^{\circ} 5$ ).

TABLA N $\mathbf{N}^{\circ}$ 5. Distribución del personal con síndrome metabólico de acuerdo a la actividad laboral

\begin{tabular}{|l|c|c|}
\hline \multicolumn{1}{|c|}{ Actividad Laboral } & Frecuencia & Porcentaje \\
\hline $\begin{array}{l}\text { Abundante actividad: liniero, } \\
\text { lector }\end{array}$ & 10 & 27,03 \\
$\begin{array}{l}\text { Moderada actividad: } \\
\text { auxiliar, técnico } \\
\begin{array}{l}\text { Escasa actividad: chofer, } \\
\text { administrativo }\end{array}\end{array}$ & 61 & 16,22 \\
Total & 37 & 100,0 \\
\hline
\end{tabular}

Fuente: Base de Datos, Personal Masculino, Empresa Eléctrica de Azogues C.A.

Si bien no existió asociación estadística entre el consumo de alcohol y valores de colesterol HDL en sangre, se encontró al relacionarla con la variable triglicéridos que en quienes refirieron nunca consumir alcohol la media fue de $188,99 \mathrm{mg} / \mathrm{dl}$ siendo menor que aquellos quienes afirmaban consumirlo con una media de $211,06 \mathrm{mg} / \mathrm{dl}$. Un hallazgo interesante fue el encuentro de la relación entre consumo de alcohol y triglicéridos séricos elevados, obteniéndose una asociación estadística significativa entre estas dos variables con $\mathrm{p}<0,05$ (Tabla No 6). 
TABLA No 6. Correlación estadística entre las variables: Consumo de alcohol y Triglicéridos.

\begin{tabular}{|c|c|c|c|c|}
\hline \multicolumn{4}{|c|}{ BEBIDAS ALCOHOLICAS } & Estadístico \\
\hline \multirow[t]{18}{*}{ Triglicéridos $(\mathrm{mg} / \mathrm{dl})$} & \multirow[t]{9}{*}{ consumo de alcohol } & Media & & 211,0638 \\
\hline & & \multirow{2}{*}{$\begin{array}{l}\text { Intervalo de confianza } \\
\text { para la media al } 95 \%\end{array}$} & Limite inferior & 176,5498 \\
\hline & & & Límite superior & 245,5778 \\
\hline & & Mediana & & 155,5500 \\
\hline & & Varianza & & 17230,114 \\
\hline & & Desv. típ. & & 131,26353 \\
\hline & & Mínimo & & 90,80 \\
\hline & & Máximo & & 630,40 \\
\hline & & Rango & & 539,60 \\
\hline & \multirow[t]{9}{*}{ nunca } & Media & & 188,9946 \\
\hline & & \multirow{2}{*}{$\begin{array}{l}\text { Intervalo de confianza } \\
\text { para la media al } 95 \%\end{array}$} & Límite inferior & 152,2654 \\
\hline & & & Límite superior & 225,7239 \\
\hline & & Mediana & & 132,3500 \\
\hline & & Varianza & & 18810,355 \\
\hline & & Desv. típ. & & 137,15085 \\
\hline & & Mínimo & & 49,70 \\
\hline & & Máximo & & 702,70 \\
\hline & & Rango & & 653,00 \\
\hline
\end{tabular}

Fuente: Base de Datos, Personal Masculino, Empresa Eléctrica de Azogues C.A.

\section{Discusión.}

En el presente estudio se encontró una prevalencia de Síndrome Metabólico del 32.5\% $(\mathrm{m}=37)$. Esta prevalencia es ligeramente superior a la encontrada en el Ecuador en población de 10 a 59 años por la encuesta ENSANUT-ECU (27,7\%) (11), y es muy similar a la prevalencia de SM encontrada en España en adultos masculinos por Fernández y Sanz (30\%) (10) y a la prevalencia del estudio Sirit realizada a la población venezolana de 26 y 40 años (de 32.1\%) (2). El grupo etario con mayor prevalencia de SM fue de 40 y 49 años (54.05\%), cifra superior a la obtenida en un estudio similar realizado en trabajadores de la Península de Santa Elena en quienes se encontró una prevalencia del $30.51 \%$ de acuerdo a criterios IDF en el año 2014 (29), se encontró una relación estadísticamente significativa entre edad y aparición de SM comprobándose en este estudio evidencias mundiales que a mayor edad mayor prevalencia de este síndrome. Cabe mencionar que en ninguna historia clínica revisada se encontró el registro diagnóstico de síndrome metabólico, lo cual apoyaría la tendencia existente en varios países en cuanto a un subregistro de esta patología que conlleva a que la mayoría de personas que padecen de la misma la desconozcan (30).

Causa preocupación el hecho de que al valorar la población sin SM aunque esta no cumplió con los criterios requeridos por la IDF para el diagnóstico de SM, existía un número importante de casos con criterios positivos siendo el más frecuente el perímetro abdominal, así como c-HDL bajo y triglicéridos elevados presentes en el 49,1\% y 48,2 \% de la 
población total respectivamente. Ambas cifras mayores que las reportadas por ENSANUTECU a nivel nacional niveles de HDL bajos en el $46.3 \%$ de la población masculina, hipertrigliceridemia del $28.7 \%$ en la población total, con un predominio en hombres con un $33.3 \%$, siendo de igual manera más preocupantes los resultados de este estudio (11). Los datos estadísticos de nuestro estudio muestran la magnitud del problema que representa el síndrome metabólico.

En cuanto a los variables del SM, durante el examen físico se identificó que el componente más frecuente para su desarrollo fue la obesidad abdominal que la presentó el 100\% de la población con este diagnóstico, encontrándose además un perímetro abdominal elevado en el $71.1 \%$ de la población total de este estudio, prevalencia mucho mayor que la encontrada en ENSANUT-ECU (68,9\% en el sexo masculino) (11).

La presencia conjunta de sobrepeso y obesidad tuvo una alta prevalencia en la población total $(76,32 \%)$ de los cuales el $64.91 \%$ presentaron obesidad abdominal, la cual es mayor que la referida en ENSANUT-ECU que reporta que la población con sobrepeso y obesidad representaba el $62.8 \%$ de la población ecuatoriana, presentándose con un mayor frecuencia entre la cuarta y quinta década de la vida (11). Es importante resaltar este dato ya que según un estudio publicado por Jaspinder se determinó a través de la Encuesta Nacional de Salud y Nutrición (NHANES) 2003-2006 que los hombres y mujeres con sobrepeso tienen de 6 y 5,5 veces más probabilidades de cumplir con los criterios de SM en comparación de personal por IMC normal, mientras que en las personas obesas las cifras incrementan bruscamente: riesgo mayor de SM entre 32 y 17 veces en hombres y mujeres respectivamente (15), es así que el presente estudio refleja esta realidad pues se encontró que la presencia de síndrome metabólico fue mayor en la población con sobrepeso con un $54.05 \%$ y en $40.54 \%$ de la población con obesidad.

En el presente estudio no se encontró una significancia estadística entre las variables relacionadas con estilo de vida (calidad de alimentación, ejercicio, actividad laboral, consumo de alcohol y tabaco) con síndrome metabólico, sin embargo, se debería manejar estos datos con precaución considerando que toda esta información fue tomada a partir de las respuestas proporcionadas por los participantes en la encuesta autoadministrada, que podría tener una limitada utilidad para medir con veracidad el estilo de vida de cada individuo, pues está sujeta a riesgos como distorsión de la realidad y ocultamiento de la información que podría subestimar las prevalencias reales, debido quizá a la falta de cultura social para contestar encuestas con información fidedigna.

Cabe destacar que la única asociación estadísticamente significativa encontrada entre los distintos componentes del SM y las variables relacionadas con estilo de vida fue entre la relación entre triglicéridos séricos y el consumo de alcohol con $\mathrm{p}<0,05$, asociación también encontrada en un metaanálisis publicada por Brien y Ronksley en el cual se analizó 44 estudios para valorar el efecto del consumo de alcohol en los marcadores biológicos asociado con el riesgo de enfermedad coronaria, en los cuales si bien reporto que el 
consumo de alcohol en pequeñas cantidades tiene un efecto cardioprotector incrementando los niveles de c-HDL, sin embargo consumo excesivo acompañado de otros factores como su combinación con bebidas azucaradas producía un aumento inminente de los niveles de triglicéridos (31). La fisiopatología que explica esta asociación es que el consumo excesivo de etanol genera cambios bioquímicos que provocan la esterificación de ácidos grasos con lo que incrementa la síntesis y secreción de la lipoproteína de muy baja densidad (VLDL), lo que genera hipertrigliceridemia (32).

\section{Conclusiones.}

- Los resultados de esta investigación comprobaron la correlación entre las variables Síndrome Metabólico y edad. Se demostró asociación estadísticamente significativa en la relación entre triglicéridos séricos y el consumo de alcohol.

- No se evidenció asociación entre factores de riesgo cardiovascular relacionados con estilo de vida y el desarrollo de síndrome metabólico, dato que hay que manejarlo con precaución considerando que la realidad podría estar enmascarada por la falta de confiabilidad en las respuestas brindadas en la encuesta.

- Además el estudio suministra informaciones para realizar investigaciones futuras y alerta para la necesidad de dar mayor atención a la salud de la población laboral y adoptar estrategias para la promoción de la salud ocupacional.

\section{Agradecimiento.}

- Los resultados de esta investigación comprobaron la correlación entre las variables Síndrome Metabólico y edad. Se demostró asociación estadísticamente significativa en la relación entre triglicéridos séricos y el consumo de alcohol.

- No se evidenció asociación entre factores de riesgo cardiovascular relacionados con estilo de vida y el desarrollo de síndrome metabólico, dato que hay que manejarlo con precaución considerando que la realidad podría estar enmascarada por la falta de confiabilidad en las respuestas brindadas en la encuesta.

- Además el estudio suministra informaciones para realizar investigaciones futuras y alerta para la necesidad de dar mayor atención a la salud de la población laboral y adoptar estrategias para la promoción de la salud ocupacional. 


\section{Referencias bibliográficas.}

Sirit Y, Acero C, Bellorin M, Portillo R. Síndrome Metabolico y otros factores de riesgo cardiovascular en trabajadores de una Planta de Policloruro de Vinilo. Rev. Salud Pública. 2008, Vol. $10 \quad$ (2): 239-249. Disponible en: http://www.scielosp.org/pdf/rsap/v10n2/v10n2a04.pdf.

Cássia E, Basei R, Avancini P, Waldomiro C Metabolic syndrome in workers in a university hospital. Rev Port Cardiol. 2012, Vol. 31 (10): 629-636. Disponible en: http://www.ncbi.nlm.nih.gov/pubmed/22981853.

Rodriguez B, Sánchez G, Campos A, Baez E, Fernandez J, Achiong F. Síndrome metabólico: un problema de salud con múltiples definiciones. Rev Colomb Cadiol. 2008, $\begin{array}{lllll}\text { Vol. } & 15 & \text { (3): } & \text { 111-26. }\end{array}$ http//www.infona.pl/resource/bwmeta1.element.elsevier-b6376c53-8413-3116-900003295ab4e54a.

Garza F, Ferreira J, Rio A, Prevención y tratamiento del síndrome metabólico. Rev Esp Cardiol Supl. 2005, Vol. 5 (1): 46-52. Disponible en: http://www.revespcardiol.org/es/prevencion-tratamiento-del-sindromemetabolico/articulo/13083448.

Ministerio de Salud Pública (MSP). [Internet]. Información estadística y geográfica de salud, producción 2014. [Citado 2015 Mayo]. Disponible en: http://salud.gob.ec/informacion-estadistica-de-produccion-de-salud/.

Lizarzaburu C. Síndrome metabólico: concepto y aplicación práctica. An Fac med. 2013, Vol. $\quad 74 \quad$ (4): $\quad 315-20 . \quad$ Disponible http://revistasinvestigacion.unmsm.edu.pe/index.php/anales/article/view/2705.

Martínez G, Alonso R, Novik V. Síndrome metabólico. Bases clínicas y fisiopatológicas para un enfoque terapéutico racional. Rev Méd Chile. 2009; Vol. 6 (137): 685-694. Disponible en: http://www.scielo.cl/scielo.php?pid=S003498872009000500014\&script=sci_abstract\&tlng=es.

Jana V, Marja L, Slagter S, Doiron D, Fischer K, Foco L, Gaye A, Gögele M, et al. Prevalence of metabolic syndrome and metabolically healthy obesity in Europe: a collaborative analysis of ten large cohort studies. BMC Endocrine Disorders. 2014, Vol. 14 (9): 1142-1186. en: https://bmcendocrdisord.biomedcentral.com/articles/10.1186/1472-6823-14-9. 
Petrella R. Associations Between Heart Rate Variability and Metabolic Syndrome Risk Factors. University of Western Ontario. 2013 Vol. 1 (8). 145-169. Disponible en: http://ir.lib.uwo.ca/cgi/viewcontent.cgi?article=2547\&context=etd.

Fernández D, Cabrera A, Sanz H, Elosua R, Guembe M, Alzamora M, et al. Síndrome metabólico en España: prevalencia y riesgo coronario asociado a la definición armonizada y a la propuesta por la OMS. Rev Esp Cardiol. 2012, Vol. 65(3): 241-248. Disponible en: http://www.revespcardiol.org/es/sindrome-metabolico-espana-prevalenciariesgo/articulo/90097768/.

Freire R, Ramírez M, Belmont P, Mendieta M, Silva M, Romero N, et al. Resumen Ejecutivo, Tomo I, Encuesta Nacional de Salud y Nutrición del Ecuador. ENSANUTECU. Ministerio de Salud Pública/Instituto Nacional de Estadísticas y Censos 2011-2013. Disponible en: http://www.ecuadorencifras.gob.ec/documentos/webinec/Estadisticas_Sociales/ENSANUT/MSP_ENSANUT-ECU_06-10-2014.pdf.

Jaspinder Kaur. A Comprehensive Review on Metabolic Syndrome. Cardiology Research and Practice. 2014. Vol. 2 (16): 1-21. Disponible en: https://www.hindawi.com/journals/crp/2014/943162/abs/.

Escobedo J, Schargrodsky H, Champagne B, Silva H, Boissonnet C, Vinueza R, et al. Prevalence of the Metabolic Syndrome in Latin America and its association with subclinical carotid atherosclerosis: The CARMELA cross sectional study. Cardiovascular Diabetology. 2009, Vol. 8(52): 534-551. Disponible en: https://cardiab.biomedcentral.com/articles/10.1186/1475-2840-8-52.

International Diabetes Federation. Metabolic syndrome. Rev. Driving the CVD epidemic. 2010. Vol. 11 (1): 1-6. Disponible en: https://www.idf.org/webdata/docs/IDF_Meta_def_final.pdf.

Schnell M, Dominguez Z, Carrera C. Aspectos genéticos, clínicos y fisiopatológicos del Síndrome Metabólico. Anales Venezolanos de Nutrición. 2007, Vol. 20 (2): 92-98. Disponible en: http://anales.fundacionbengoa.org/ediciones/2007/2/art5.pdf.

Garcia E. La obesidad y síndrome metabólico un problema de salud pública. Revista Salud Pública México. 2008. Vol. 1 (50): 530-557. Disponible en: http://www.scielo.org.mx/pdf/spm/v50n6/15.pdf.

53.o Consejo Directivo 66. Sesión del comité regional de la OMS para las Américas. Plan de acción para la prevención de la obesidad en la niñez y la adolescencia. Washington, D.C. ECU, 2014, Vol. 1 (1):1-15. Disponible en: http://www.paho.org/hq/index.php?option=com_content $\& v i e w=\operatorname{article} \& i d=9774 \% 3 \mathrm{~A} 2014$ -53rd-directing-council\&catid=8811\%3Adc-documents \&Itemid=41062\&lang=es. 
Kassi E, Pervanidou P, Kaltsas G, Chrousos G. Metabolic syndrome: definitions and Controversies. BMC Medicine. 2011, Vol. 9(48): 145-163. Disponible en: http://bmcmedicine.biomedcentral.com/articles/10.1186/1741-7015-9-48.

Diabetes Voice. Sindrome Metabólico. International Diabetes Federation. 2006. Vol. 1(51): 2-37. Disponible en: https://www.idf.org/sites/default/files/attachments/issue_43_es.pdf.

Pineda Carlos. Síndrome metabólico: definición, historia, criterios. Colomb Med. 2008, Vol. 1(39): 96-106. Disponible en: http://www.bioline.org.br/pdf?rc08013.

Angelini J. “¿Existe correlación entre los valores del Perímetro Abdominal y el equilibrio metabólico?”. Universidad Nacional de La Plata, Facultad de Ciencias Médicas. Buenos Aires-Argentina. 2010, Vol. 1 (1):1-19. Disponible en: http://sedici.unlp.edu.ar/discover?fq=type_filter\%3Atrabajol+del+especializacion ||||$T r a b a j$ o\+del+especializacion.

Lahsen R. Síndrome Metabólico y Diabetes. Revista Médica. Clinica. Condes. 2014, Vol. 1(25) 47-52. Disponible en: http://www.sciencedirect.com/science/article/pii/S0716864014700100.

Singh Y. Tobacco and metabolic syndrome. Indian J Endocrinol Metab. 2012 Vol.1 (16): 81-87. Disponible en: http://www.ncbi.nlm.nih.gov/pmc/articles/PMC3263202/.

The Institute of Human Genomic Study Republic of Korea. Prospective study of alcohol consumption and metabolic syndrome. American Society for Clinical Nutrition. 2008, Vol. (1): 1-6. Disponible en: http://www.ncbi.nlm.nih.gov/pubmed/18469271.

Sistema de Vigilancia Epidemiológica Sive-Alerts. Tasa de consumo de alcohol por provincia y región. Ministerio de salud Pública. 2011, Vol. 1(1):1-13 Disponible en: http/www.salud.gob.ec/.

Consejo Nacional de Control de Sustancias Estupefacientes y Psicotrópicas - CONSEP. Tercera encuesta nacional sobre: Consumo de drogas en estudiantes de enseñanza media 2008, Vol. 1(1): 3-23. Disponible en: http://www.prevenciondrogas.gob.ec/wpcontent/uploads/2015/08/Tercera-Encuesta-Nacional-sobre-uso-de-drogas-en-estudiantesde-ense $\%$ C3\%B1 anza-media.pdf.

Página web. Organización Mundial de la Salud: http://www.who.int/mediacentre/factsheets/fs394/es/.

Bernhardi R, Zanlungo S, Arrese M, Arteaga A, Rigotti A. El síndrome metabólico: De factor agravante a principal factor de riesgo patogénico en diversas enfermedades crónicas. Rev Med Chile 2010, Vol. 1(138): 1012-1019. Disponible en: http://www.scielo.cl/scielo.php?script=sci_arttext\&pid=S0034-98872010000800012. 
Aguirre M, Crespo P. Prevalencia de Síndrome Metabólico Adultos. Tesis de Grado previa a la obtención de título de médico, Universidad del Azuay, 2015: En los trabajadores de la Península de Santa Elena. Revista Científica y Tecnológica UPSE. Disponible en: http//dspace.uazuay.edu.ec/bistream/datos/4217/10778.pdf.

Reyes M, Benitez N, Lapertosa S. Frecuencia del síndrome metabólico en pacientes con diabetes mellitus tipo II en un servicio de diabetes de Corrientes. Revista de Postgrado de la VIa Cátedra de Medicina. 2008, Vol. 1(185), 23-57. Disponible en: http//med.unne.edu.ar/revista/revista185/2_185.pdf.

Brien S, Ronksley P, Turner B, Mukamal K, William A. Effect of alcohol consumption on biological markers associated with risk of coronary heart disease: systematic review and meta-analysis of interventional studies. British Medical Journal. 2011, Vol. 1(1): 342-636. Disponible en: http://www.bmj.com/content/bmj/342/bmj.d636.full.pdf.

Rosa E, Laguna J. Tratamiento de la hipertrigliceridemia: fibratos frente a ácidos grasos omega-3. Revista Española de Cardiología. 2006, Vol. 1(6): 52-61. Disponible en: http://www.revespcardiol.org/es/tratamiento-hipertrigliceridemia-fibratos-frenteacidos/articulo/13091599/. 


\section{Para citar el artículo indexado.}

Suárez K. \& Vinueza M. . (2018). Prevalencia de síndrome metabólico y factores de riesgo cardiovascular en el personal masculino de la Empresa Eléctrica de Azogues C.A periodo abril-noviembre 2015. Revista electrónica Ciencia Digital 2(3), 228-242. Recuperado desde:

http://cienciadigital.org/revistacienciadigital2/index.php/CienciaDigital/article/view/149/13 $\underline{4}$

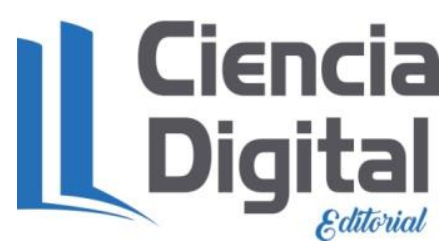

El artículo que se publica es de exclusiva responsabilidad de los autores y no necesariamente reflejan el pensamiento de la Revista Ciencia Digital.

El articulo queda en propiedad de la revista y, por tanto, su publicación parcial y/o total en otro medio tiene que ser autorizado por el director de la Revista Ciencia Digital.
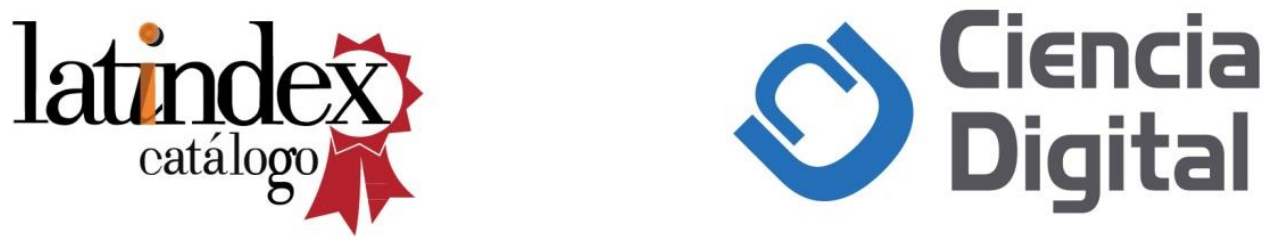